\title{
On the statistical detection of propagating waves in polar coronal holes
}

\author{
G. R. Gupta ${ }^{1,2}$, E. O’'Shea ${ }^{3}$, D. Banerjee ${ }^{1}$, M. Popescu ${ }^{3}$, and J. G. Doyle ${ }^{3}$ \\ 1 Indian Institute of Astrophysics, Bangalore 560034, India \\ e-mail: girjesh@iiap.res.in \\ 2 Joint Astronomy Programme, Indian Institute of Science, Bangalore 560012, India \\ 3 Armagh Observatory, College Hill, Armagh BT61 9DG, N. Ireland
}

Received 15 July 2008 / Accepted 23 October 2008

\begin{abstract}
Context. Waves are important to the study of dynamical processes in coronal holes and the acceleration of the fast solar wind. A spectral time series was taken with the SUMER spectrometer on-board SoHO on 20 October 1996. The observations were obtained in the N IV $765 \AA$ transition region line and the Ne VIII $770 \AA$ line of the low corona.

Aims. We detect the presence of waves and study their characteristic properties in terms of their propagation speeds and direction. Previous statistical studies, undertaken with data from the CDS spectrometer, report the presence of waves in these regions. We extend this analysis using SUMER observations.

Methods. Using Fourier techniques, we measured the phase delays between intensity oscillations, as well as between velocity oscillations, in our two lines over the full range of available frequencies. From this, we were able to measure the travel time of the propagating oscillations, hence the propagation speeds of the waves that produce the oscillations.

Results. We detect the long period oscillations in polar coronal holes on the disc. For network bright locations within coronal holes, our results indicate the presence of compressional waves with a dominant period of $\approx 25 \mathrm{~min}$. However, we also find power at many other different frequencies, so we are able to study oscillations over a full range of frequencies. We find evidence of propagating waves with a fixed time delay in the coronal hole. We find, moreover, that there is a difference in the nature of the wave propagation in the bright ("network"), as opposed to the dark ("internetwork") regions, with the latter sometimes showing evidence of downwardly propagating waves that are not seen in the former. From a measurement of propagation speeds, we find that all measured waves are subsonic in nature.

Conclusions. Waves with different characteristics are found to be present at different locations in the observed coronal hole. The measured propagation speeds are subsonic, indicating that the majority of them are slow magneto-acoustic in nature. These waves, measured in the lower atmosphere, could accelerate farther at higher altitudes and may be important for the acceleration of the fast solar wind.
\end{abstract}

Key words. Sun: corona - Sun: oscillations - Sun: UV radiation - Sun: transition region - waves

\section{Introduction}

Coronal holes are regions of cool and low density plasma that are "dark" at coronal temperatures (Munro \& Withbroe 1972). During the years of solar minimum, coronal holes are confined to the Sun's polar regions, while at solar maximum they can also be found at lower latitudes, usually associated with remnant active regions, as so-called "equatorial" coronal holes (Timothy et al. 1975). The predominantly unipolar magnetic field from coronal hole regions is thought to give rise to the fast solar wind (Krieger et al. 1973).

The prominent feature of the coronal holes in the chromosphere and transition region is the magnetic network, which is believed to be the upward extension of the supergranular boundaries above the photosphere (Reeves 1976). The network is identified as bright lanes in radiance images, the darker areas within the network cells are termed as internetwork. It is suggested that part of the network fans out in the corona into a funnel shape. Recently, it has been further suggested that the fast solar wind originates from coronal hole funnels (Tu et al. 2005). Different studies have found evidence for outflows (blue shifts) of typically $10 \mathrm{~km} \mathrm{~s}^{-1}$ in both polar (Wilhelm et al. 2000;
Popescu et al. 2004) and equatorial (Xia et al. 2004) coronal holes at transition region temperatures.

A number of studies have measured oscillations in coronal holes in the polar off-limb regions of the Sun (Ofman et al. 1997, 2000; Banerjee et al. 2001; Popescu et al. 2005). All of these studies point to the presence of compressional waves, thought to be slow magneto-acoustic waves as found by DeForest \& Gurman (1998), O'Shea et al. (2006, 2007).

In this work, we look for additional evidence of waves in the disc part of polar coronal hole regions by carrying out a statistical study of oscillations and their phase properties, both in the intensity and velocity domain. The observations were carried out with the SUMER spectrometer (solar ultraviolet measurements of emitted radiation) on-board the solar and heliospheric observatory (SoHO) spacecraft (Wilhelm et al. 1995). We will focus on the behaviour of oscillations detected in the bright network regions and the darker internetwork regions.

\section{Observations and data analysis}

The data selected for this study were obtained as a time series on 20 October 1996, starting at 19:57 UT, in a Northern polar 


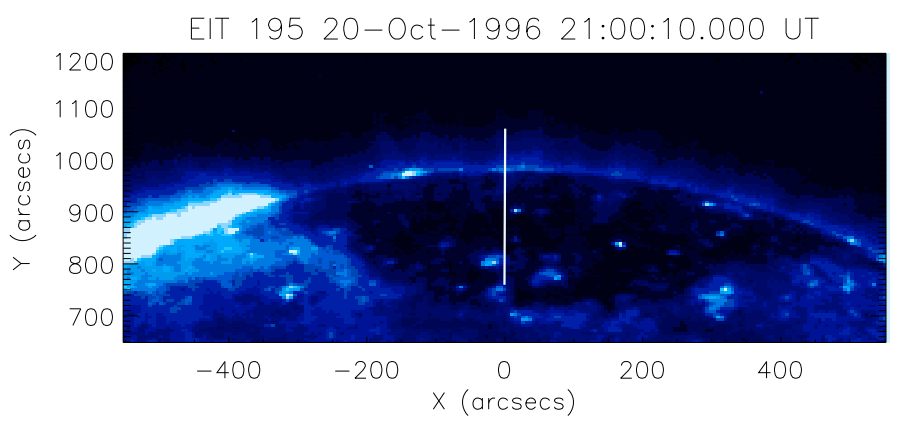

Fig. 1. Solar polar coronal holes imaged by EIT/SoHO in the $195 \AA$ band. The vertical white lines show the fixed position of the SUMER slit.

coronal hole (hereafter $\mathrm{pCH}$ ) as observed by SUMER with the $1^{\prime \prime} \times 300^{\prime \prime}$ slit using an exposure time of $30 \mathrm{~s}$. The two lines used in this study are the transition region N IV $765 \AA$ line at a temperature of $\log T=5.0 \mathrm{~K}(T=100000 \mathrm{~K})$ and the Ne VIII $770 \AA$ coronal line at a temperature of $\log T=5.8 \mathrm{~K}(T=630000 \mathrm{~K})$. During the observation, the SUMER slit was kept fixed at the north pole $\left(X=0^{\prime \prime}, Y=909.75^{\prime \prime}\right)$ (see Fig. 1) with part of the slit off-limb and part in the disc part of the coronal hole (sitand-stare observations). Figure 1 shows the initial position of the SUMER slit overplotted on an EIT (the extreme ultraviolet imaging telescope on the SoHO spacecraft) image taken in the $195 \AA$ filter. The pCH can be clearly identified in the image as very dark regions of reduced coronal emission at the poles. The slit width $\left(1^{\prime \prime}\right)$ gives the spatial resolution along the $x$-direction, whereas the resolution along the slit in the $y$-direction (northsouth; positive towards north) is given by the pixel size of the detector, and is also approximately $1^{\prime \prime}$. For sit-and-stare observations one needs to consider the effect of the solar rotation on the detectable minimum frequency. We used the program, rot_xy.pro within solarsoft ${ }^{1}$, which incorporates diff_rot.pro ${ }^{2}$, to calculate the rotation rate. The diff_rot.pro procedure uses standard equations for calculating solar rotation, while rot_xy.pro converts the results of this from degrees/day to arcsec/day (second). At the lowest pixel location, $Y \approx 760^{\prime \prime}$, the rotation rate is $\approx 3.7^{\prime \prime} / \mathrm{h}$. Thus the highest frequency that can possibly be affected by rotation is $\approx 1.03 \mathrm{mHz}$ (Doyle et al. 1998). Whereas at high latitudes $\left(Y=950^{\prime \prime}\right.$ and higher) the solar rotation is very slow $\left(\leq \approx 0.255^{\prime \prime} / \mathrm{h}\right)$ and thus the spreading of the frequencies is negligible $(\leq \approx 0.071 \mathrm{mHz})$. In general, most frequencies are measured from pixels above $Y \approx 760^{\prime \prime}$ and, thus, the effects of rotation will be at values much less than $\approx 1.03 \mathrm{mHz}$.

We applied the standard procedures for correcting and calibrating the SUMER raw data, namely decompression, reversal as well as flat field, dead-time, local-gain, and geometrical corrections ${ }^{3}$. The method used to deduce the line parameters (radiance, central position of the spectral line and width) is described as follows. We calculated the central position for every pixel, by integrating the line radiance across a certain spectral window and determining subsequently the location of the $50 \%$ level with sub-pixel accuracy. This procedure is frequently used to obtain SUMER Dopplergrams (see details in Dammasch et al. 1999)

\footnotetext{
1 http://www .astro. washington . edu/deutsch-bin/getpro/ library32.html?ROT_XY()

2 http://www.astro.washington.edu/deutsch-bin/getpro/ library32.html?DIFF_ROT()

3 http://www.ias.u-psud.fr/website/modules/ content_sol/index.php?id=1068
}

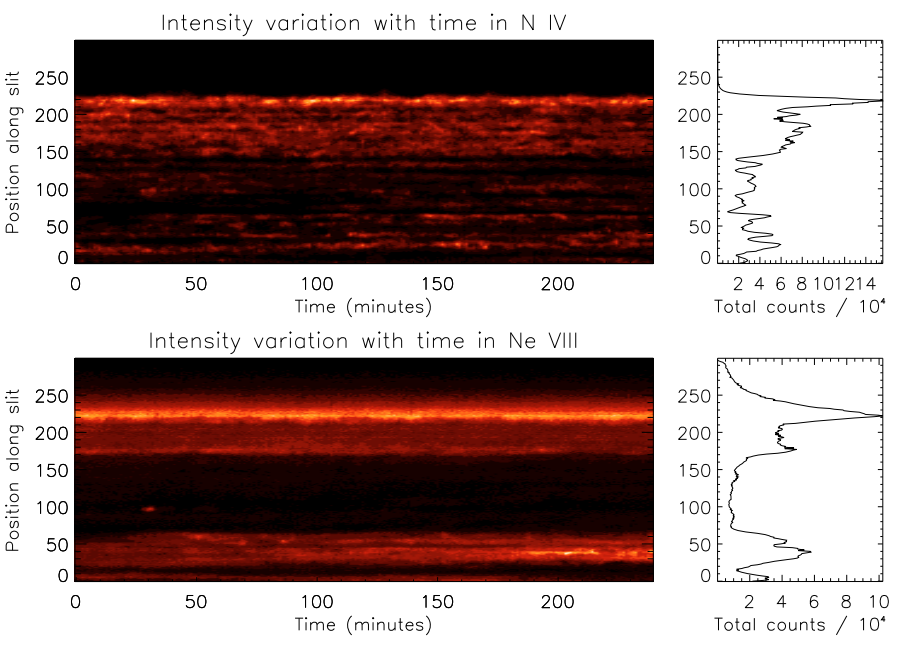

Fig. 2. Top (bottom) left panel shows the variation of the N IV $765 \AA$ (Ne VIII $770 \AA$ ) intensity along the slit (in pixel units) with time. Right panel shows the variation of the total summed counts over time along the slit.

and dramatically reduces the computing time for a large number of data. The results deduced by this method are statistically consistent with those obtained by using the standard Gaussian fitting programme (Xia et al. 2003). In addition, a line-position correction was applied, to remove spurious spectral line shifts caused by thermal deformations of the instrument, and to eliminate residual errors (systematic variation along the slit) after the geometric correction, using the standard software (Dammasch et al. 1999; Xia et al. 2003). The uncertainty in velocity from this calculation is $\pm 1.2 \mathrm{~km} \mathrm{~s}^{-1}$ (Dammasch et al. 1999). The line of sight (LOS) velocity values presented in this paper are measured relative to the limb velocity, i.e., the velocity at the limb is assumed to be effectively zero. We binned over two time frames and two spatial pixels to increase the signal to noise, which yields an effective cadence of $60 \mathrm{~s}$. The Nyquist frequency, therefore, is $\approx 8.33 \mathrm{mHz}$ (Jenkins \& Watts 1968). The total observation time is $240 \mathrm{~min}=1.44 \times 10^{4} \mathrm{~s}$ and, hence, the frequency resolution is $\approx 6.94 \times 10^{-2} \mathrm{mHz}$. In the following Fourier analysis, phase delays will be measured at all the frequencies up to the Nyquist frequency, at steps dictated by the frequency resolution.

The Quiet Sun (QS) chromosphere and transition region show a network pattern all over the disc, with intensity enhancements in the network boundaries. Within coronal holes such patterns also exist (Tu et al. 2005). Presumably, the magnetic field is predominantly concentrated on such network boundaries and, within coronal holes, the footpoints of coronal funnels emanate from these network regions. Recent studies from SOT/HINODE also indicate that these funnels originate from small magnetic patches with very strong $\mathrm{kG}$ magnetic fields (Tsuneta et al. 2008). Tsuneta et al. (2008) further claims that all the open field lines forming the polar coronal hole essentially originate from such scattered small but intense magnetic patches, and the fast solar winds emanate from these vertical flux tubes seen in the photosphere. We assume that our intensity enhanced regions could correspond to these magnetic patches. Thus, to identify such locations and distinguish between network and internetwork pixels, the subsets of data to be used in our study were selected on the basis of bright ("network") and dark ("internetwork") locations. The left panels of Fig. 2 show the variation of the N IV (top panel) and the Ne VIII (lower panel) intensity along the slit with time. The right panels show the variation of the total 

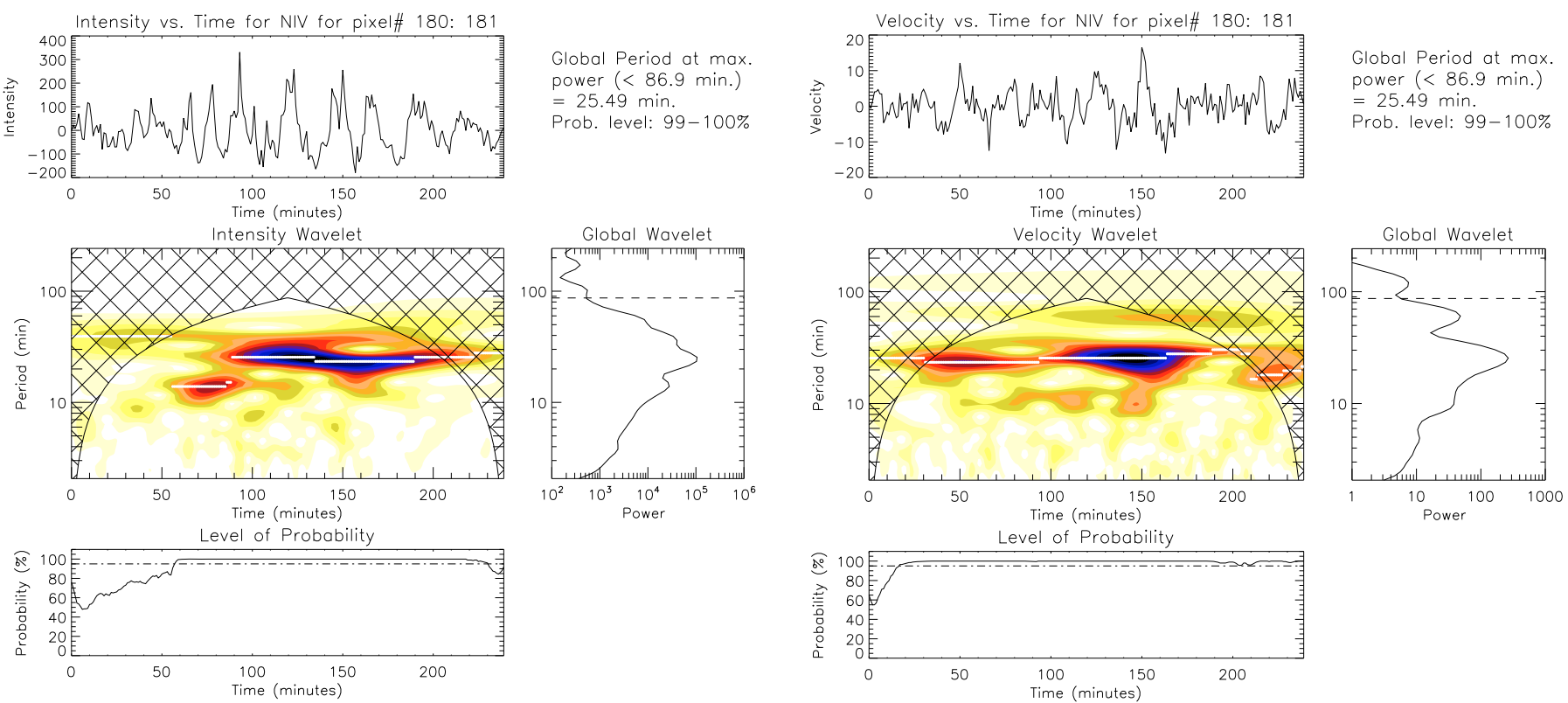

Fig. 3. The wavelet result for a bright location (pixel No. $=180-181$ which corresponds to $Y \approx 940^{\prime \prime}$ ) in the N IV $765 \AA$ intensity and velocity. In each set the top panels show the relative (background trend removed) radiant flux/velocity as marked, the central panels show the colour inverted wavelet power spectrum, the bottom panels show the variation of the probability estimate associated with the maximum power in the wavelet power spectrum (marked with white lines), and the right middle panels show the global (averaged over time) wavelet power spectrum. Above the global wavelet the period, measured from the maximum power from the global wavelet, together with probability estimate, is printed.

summed counts over time at each pixel position along the slit. The intensity enhanced locations marks the location of network boundary. For choosing the bright ("network") locations, we have used the arbitrary criterion that pixels having an intensity higher than 1.35 times the average intensity ( $\sim 2000$ counts) are network pixels (excluding those very close to the limb and offlimb pixels, beyond $\approx 210$ pixels). This corresponds to the intensity enhancements in the right top panel of Fig. 2 and their nearest neighbours. The pixels numbered $23-26$ and $\approx 149-200$ are identified as bright pixels and other pixels as dark (internetwork) for the low temperature N IV line (note that in this particular case the network boundary spans a large spatial domain along the slit, maybe it is a bigger patch as seen by recent HINODE studies). The network pixels obtained from N IV line are assumed to be the same in the higher temperature Ne VIII line.

\section{Results and discussions}

In Figs. 3 and 4, we show a representative example of the type of the oscillations we measured in a bright region of the $\mathrm{pCH}$ at the pixel location 180-181 which corresponds to the solar- $Y$ location of $\approx 940^{\prime \prime}$. At this location the rotation rate is $\approx 0.87^{\prime \prime} / \mathrm{h}$. Thus the highest frequency that can possibly be affected by rotation is $\approx 0.24 \mathrm{mHz}$. Figure 3 shows the variation of the intensity (hereafter we will use the term intensity for trend subtracted radiant flux) and relative velocity (trend subtracted), as a function of time. Details on the wavelet analysis, which provides information on the temporal variation of a signal, are described in Torrence \& Compo (1998). For the convolution with the time series in the wavelet transform, we chose the Morlet function, as defined in Torrence \& Compo (1998). The oscillations shown in the upper panel had their background trend removed by subtracting from the original time series a 30-point running average (i.e., $\mathrm{a} \approx 30$ min interval). In the wavelet spectrum, the cross-hatched regions are locations where estimates of oscillation period become unreliable. This is the so-called cone-of-influence (COI), see Torrence \& Compo (1998). As a result of the COI, the maximum measurable period is shown by a dotted line in the global spectrum plots. Above the global wavelet spectrum of Fig. 3 is shown the period, measured at the location of the maximum in the global wavelet spectrum, together with an estimate of the probability that this oscillation is not due to noise. The probability estimate was calculated using the randomisation method with 200 permutations as outlined in detail in O'Shea et al. (2001). A randomisation test is based on the assumption that, if there is no periodic signal in the time series data, then the measured values (intensity, velocity, etc.) are independent of their observation times. For example, the intensities $I_{1}, I_{2}, \ldots I_{n}$, observed at times $t_{1}, t_{2}, \ldots t_{n}$, are just as likely to have occurred in any other order $I_{r(1)}, I_{r(2)}, \ldots I_{r(n)}$, where $\mathrm{n}$ is the total number of observations and $r(1), r(2), \ldots r(n)$ is a random permutation of the subscripts $1,2, \ldots$ $n$. Only those oscillations with a probability $>95 \%$ are considered to be significant. Below the wavelet power spectrum, in the lower panels, we show the variation of the probability estimate, calculated using the randomisation technique, associated with the maximum power at each time in the wavelet power spectrum. The location of the maximum power is indicated by the overplotted white lines. We should also point out that there are 240 points in the intensity time series, with a separation of $60 \mathrm{~s}$, which result in a range of (non-linear) period scales, or values, given by the wavelet. These scales (or period values) from the wavelet are at fixed values of period and so small variations of period between one oscillation period and another can be lost. In our case, in Figs. 3 and 4, we see periods at a reduced range of values of 25.49 and $23.38 \mathrm{~min}$. It is most probable that the oscillations have a range of values between 23-26 min. However, their precise values must remain unknown due to the relative crudity of the temporal resolution given by the wavelet scales. Thus we can see oscillations of $\approx 25$ min period in both the intensity and the velocity. So, we may say that these oscillations are likely to be present due to the propagation characteristics of a compressional wave. These oscillations can be seen in both lines (N IV 

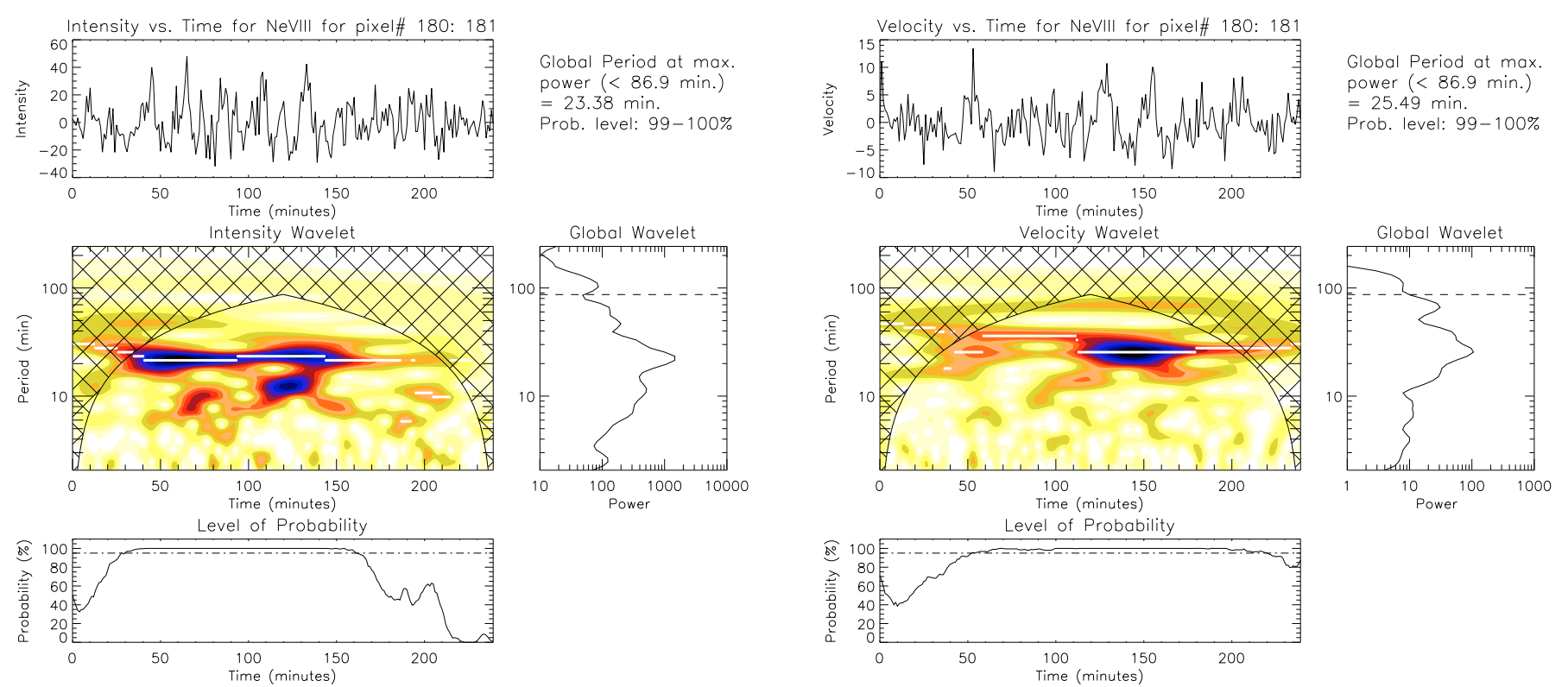

Fig. 4. The wavelet analysis results for a bright location (pixel No. $=180-181$ which corresponds to $Y \approx 940^{\prime \prime}$ ) in the intensity (left side) and in velocity (right side) data for the Ne VIII 770 A line. The descriptions about the different panels are the same as in Fig. 3.

and Ne VIII), cf. Figs. 3 and 4. We note that the fact we see a similar periodicity in the intensity and velocity wavelets is a sign of "correlation" at the strongest period of oscillation. As the oscillations are seen in both the lower and the higher temperature lines, it is likely that the waves producing these oscillations are propagating between the different temperature regions where these lines form.

To investigate whether this is actually the case, we measure the phase delays in intensity and also in the LOS relative velocity between both lines for each of the measurable pixels along the slit. For all separate frequencies the "correlation" is determined by the squared coherency spectral estimate, which is a function of frequency, with values between 0 and 1 that indicates how well a time series $x$ corresponds to a time series $y$ at each frequency. The phases are calculated from cross power spectral estimates, following the techniques outlined in the appendix of Doyle et al. (1999). Only phases (corresponding to each spatial pixel) where the squared coherencies are greater than a significance of $95.4 \%(2 \sigma)$ are used in the analysis (see O'Shea et al. 2006, and references therein). The errors in the phase is calculated based on Eq. (A23) of Doyle et al. (1999). In this work we follow the treatment used by Athay \& White (1979) and O'Shea et al. (2006), in which the calculated phase delays are plotted over the full -180 to $+180\left(360^{\circ}\right)$ range and as a function of the measured oscillation frequency. Since the expected phase delay or difference is given by the equation:

$$
\Delta \phi=2 \pi f T
$$

where $f$ is the frequency and $T$ the time delay in seconds, the phase difference will vary linearly with $f$, and will change by 360 over frequency intervals of $\Delta f=1 / T$. This will give rise to parallel lines in $\Delta \phi$ vs. $f$ plots at fixed frequency intervals $(\Delta f=1 / T)$, corresponding to a fixed time delay $T$. Following the techniques outlined in O'Shea et al. (2006), we show in Fig. 5, the combined phase delay results for bright and dark regions. The phase here is calculated for the line pair N IV $765-\mathrm{Ne}$ VIII 770 in intensity and in relative velocity. If the oscillations are due to magnetoacoustic waves, it is to be expected that wave signatures will be present in both intensity and velocity. For this reason, the calculated phases for both of these quantities are plotted together, which also helps to improve the statistics. The phases from radiant flux oscillations are shown as the grey circle symbols, while those from LOS velocities are shown as the black circle symbols. We should point out here that we have approximately 50 network and 50 internetwork pixels after binning. Each panel of Fig. 5 has about 450 phase points, including intensity and velocity phase points. On average, for each pixel position (along the slit) we have about 9 phase points, that have a significance of $95.4 \%$ and above, with more velocity points as compared to intensity points. We can see immediately that the phases in Fig. 5 are distributed between -180 and 180 . If there are fixed time delays present between the oscillations in the different lines, as in O'Shea et al. (2006), then we might expect the phases to line up along inclined parallel lines as predicted by the phase equation (Eq. (1)). We briefly summarise the method followed here. In the phase plot (Fig. 5), we first identify the location where the strong clustering of phase points forming the straight line takes place e.g., a clear line of points between 7 and $8 \mathrm{mHz}$ in the left panel of Fig. 5. This line need not pass through the origin i.e. point where the phase equals zero for $f=0$ as done in the previous studies (O'Shea et al. 2006, 2007). We note, however, that the first line does pass through this point, as expected. (For the plot in the right-hand panel, showing mostly downward propagating waves, the first line does not pass through the $f=0$ point). If we imagine, however, a further line to the left of the first line, with the same spacing in $f$, then this would likely pass through the zero point, assuming that the errors in the phases are $\approx 50-60$ degrees. After identifying the location, a straight line (between $7-8 \mathrm{mHz}$ in the left-hand plot) is fitted to these phase points. After fitting this line, all the phase points are shifted by a fixed amount which is determined from the slope of the fitted line, resulting in a transfrom which make the lines horizontal. Finally histograms are produced with these results (Fig. 6). Numerous peaks are present in the histogram. Now to check if these peaks are statistically significant or just due to a random distribution, we applied the Monte-Carlo technique, where the phase data were randomised 5000 times, to give estimates of the "noise" 

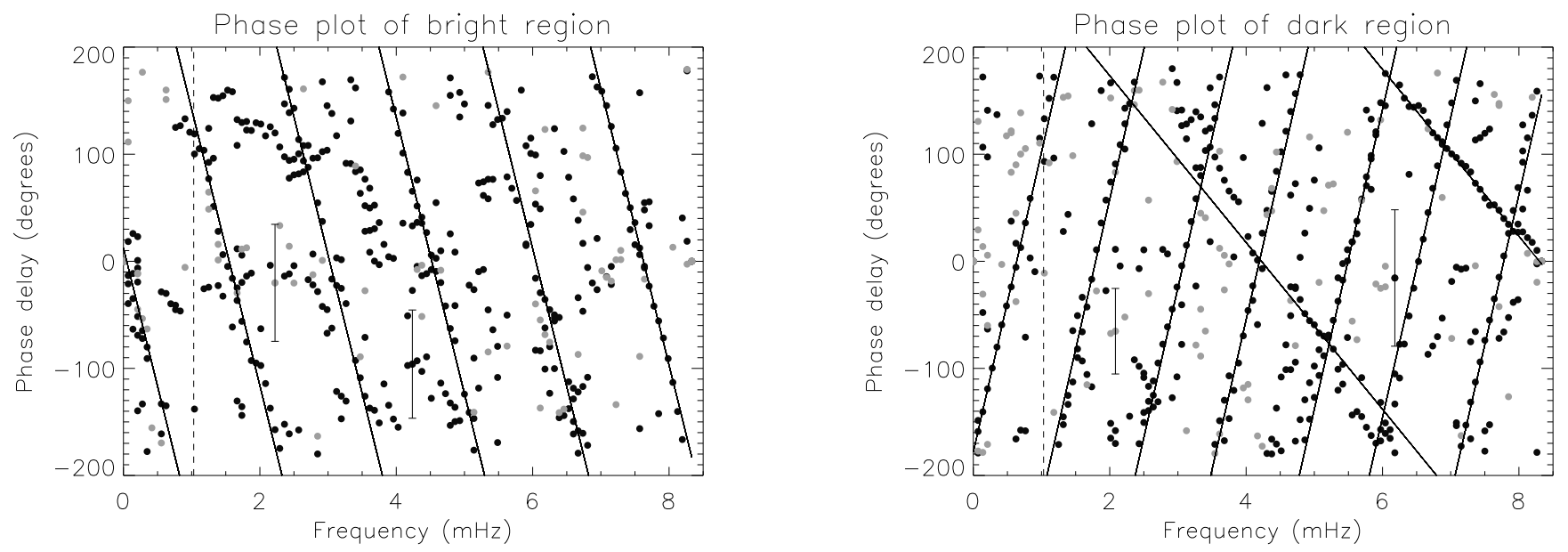

Fig. 5. Phase delays measured between the oscillations in the spectroscopic line pair for the bright (left panel) and dark (right panel) locations. The phases in radiant flux oscillations are shown in the grey circle symbols while that in LOS velocities are shown as the black circle symbols. Overplotted on each figure are black parallel lines, corresponding to fixed time delays. The vertical dashed line drawn at $1.03 \mathrm{mHz}$ indicates that some phase values below this could be affected by solar rotation. Representative errors on the phase measurements are indicated by the error bars.
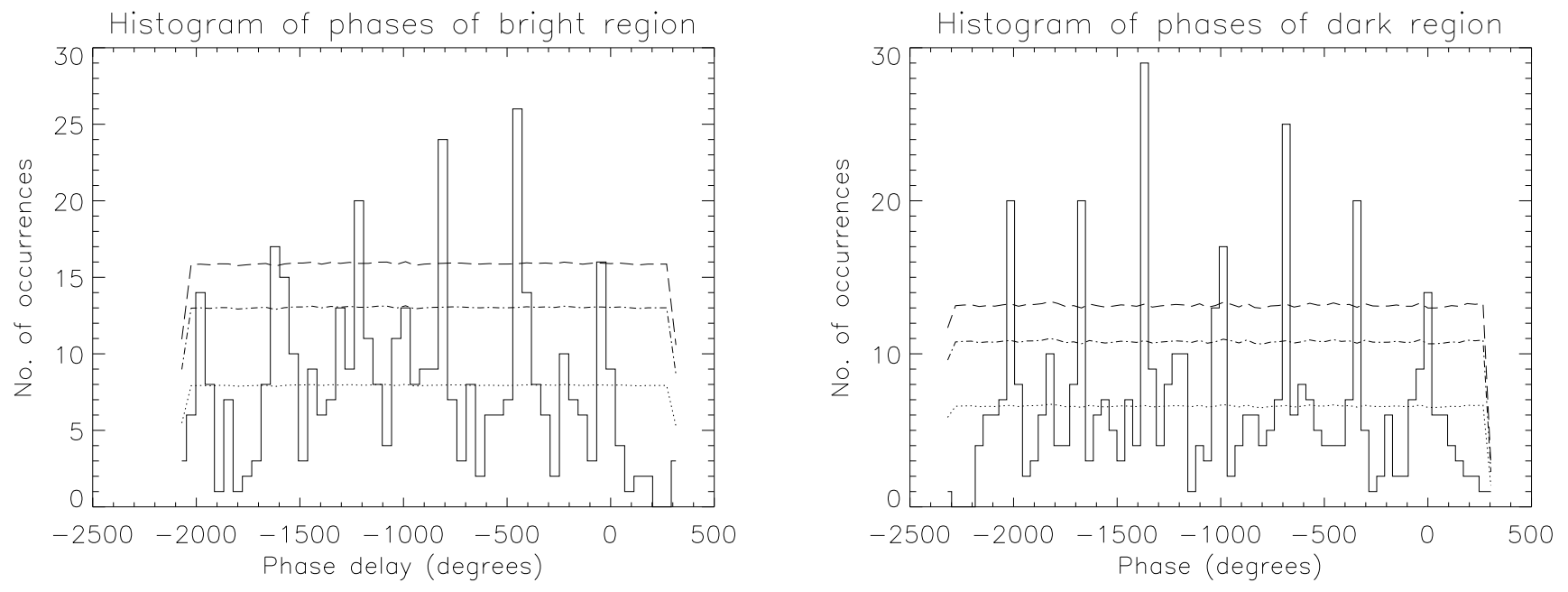

Fig. 6. Histogram showing the distribution of phase delay measurements as a function of frequency for the bright (left panel) and dark (right panel) locations for the downwardly propagating waves only. The horizontal dotted, dot-dashed and long dashed lines show the $68.3 \%$ (i.e., $1 \sigma$ ), $90 \%$ $(\approx 1.64 \sigma)$ and $95 \%(\approx 2 \sigma)$ confidence levels, respectively, calculated using Monte-Carlo simulations with 5000 permutations.

$(1 \sigma)$ level in the histogram. We have then calculated the $90 \%$ and $95.4 \%$ significance levels. So in the histogram, peaks above the $95.4 \%$ level are statistically more significant. Furthermore, each of these peak indicates the presence of a straight line at that position. Hence, other parallel straight lines are drawn in the phase plot in Fig. 5 by using these positions. Thus, by fitting lines to the phase measurements in the phase plots, we estimate the slopes and therefore the time delays between the line pair for the bright and dark regions. Looking at the left panel of Fig. 5 first, for the bright ("network") locations, it is clear that parallel lines are present in the data. These lines of phase are more obvious in the measurements made from the velocity oscillations. The implications of this result will be dealt with in the discussion section. The slope of these lines gives a time delay of $-717 \pm 114 \mathrm{~s}$. It is noticeable that these histogram peaks are constantly spaced at $\approx 400^{\circ}$. As the errors in the phases shown in Fig. 5 are of the order of $\approx \pm 55^{\circ}$, it is likely that what we see here is really just the expected variation of phases over $360^{\circ}$ for upwardly propagating waves. The consistent variation of $\approx 400^{\circ}$ suggests, as in O'Shea et al. (2006, 2007), that we may also be seeing a signature of cavity resonance. The result can indeed be interpreted as:

$\Delta \phi=2 \pi(f \pm n \Delta f) T$

where $n$ is the order of the frequency shift, i.e., $0,1,2$, etc., and $\Delta f$ is $f / 9$ for the results found here. For example, for the time delay of $-717 \mathrm{~s}$ (equivalent to a $f$ of $1.39 \mathrm{mHz}$ ), this shift, $\Delta f$, of $f / 9$ would give an exact phase spacing of $400^{\circ}$.

In the dark locations (the "internetwork") (right panel of Fig. 5), we find two sets of parallel straight lines with opposite slope. The presence of parallel lines with opposite slope indicates the presence of both upward and downward propagating waves. This is the first time, to our knowledge, that this has been reported in the literature. The measured time delays in this location are $778 \pm 133 \mathrm{~s}$ (downwardly propagating), with the lines sloping towards the right, and $-216 \pm 37.8 \mathrm{~s}$ (upwardly propagating), with the lines sloping towards the left. In right panel of Fig. 6 we plot the histograms corresponding to the downwardly 


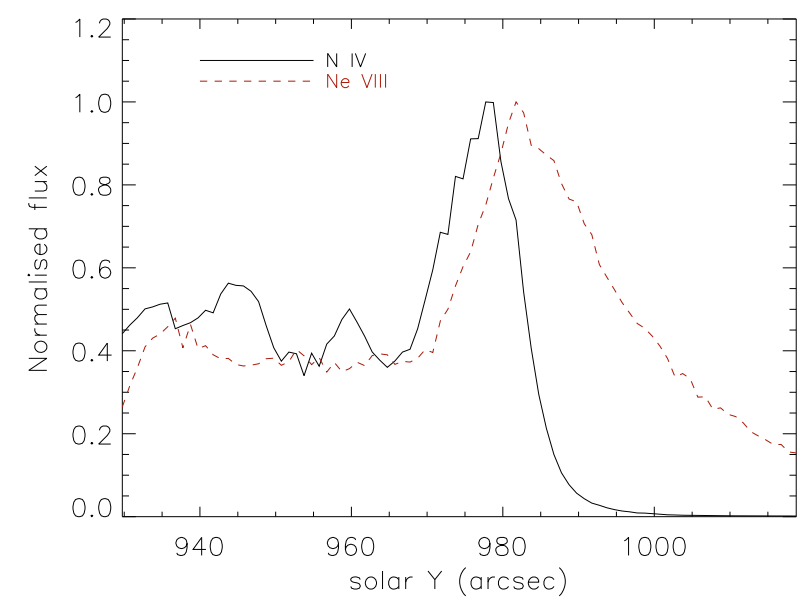

Fig. 7. Normalised flux distribution with height for the two lines. The solid line shows the distribution for N IV, while the dashed line shows the distribution for Ne VIII. The location of maximum brightness for each line is used for determining the height difference.

Table 1. Propagation speeds measured in the polar coronal hole.

\begin{tabular}{|c|c|c|c|c|}
\hline \multirow{2}{*}{$\begin{array}{c}\text { Height } \\
\text { Diff. }(\mathrm{km})\end{array}$} & \multicolumn{2}{|c|}{ Delay time (s) } & \multicolumn{2}{|c|}{ Wave speed $\left(\mathrm{km} \mathrm{s}^{-1}\right)$} \\
\hline & Bright location & Dark location & Bright location & Dark location \\
\hline \multirow{2}{*}{2860} & \multirow{2}{*}{$-717.0 \pm 114.1$} & $778.0 \pm 133.5$ & \multirow{2}{*}{$-4.0 \pm 0.6$} & $3.7 \pm 0.5$ \\
\hline & & $-216.4 \pm 37.8$ & & $-13.2 \pm 2.3$ \\
\hline
\end{tabular}

propagating waves. Similar histograms have also been studied for upward propagation but due to the lack of "lines" present in the phase no conclusions could be drawn. Notice that in the right panel of Fig. 6 the histogram peaks are all above the 95\% significance level, indicating that it is very unlikely that the lines of phase are due to noise. Notice also that the spacing of the histogram peaks is not constant, but varies between $\approx 300-340^{\circ}$. We suggest that this is the expected $360^{\circ}$ phase difference for unimpeded downwardly propagating waves, modified by the errors of $\approx \pm 55^{\circ}$ in the phase measurements.

The measured time delays may be used to estimate propagation speeds for the waves assumed to be causing the oscillations. In order to calculate the propagation speeds, one needs information on the height difference in the atmosphere between the different lines in the line pair. As described in O'Shea et al. (2006), we studied the variation of the summed flux over time along the slit position near the limb (see Fig. 7). Noting the peak intensity location we have estimated the difference in their formation heights. So using this height difference and the measured time delays, we show in Table 1 the resulting calculated propagation speeds. The sound speed in the solar atmosphere can be expressed as function of temperature alone (Priest 1984) by the equation:

$C_{\mathrm{s}}=152 T^{1 / 2} \mathrm{~m} \mathrm{~s}^{-1}$

where $T$ is the temperature in K. From this, and using the formation temperature of the lines, we can estimate the sound speeds to be $48 \mathrm{~km} \mathrm{~s}^{-1}$ at the temperature of $\mathrm{N} \mathrm{IV}\left(T \approx 1 \times 10^{5}\right)$ and $120 \mathrm{~km} \mathrm{~s}^{-1}$ at the temperature of Ne VIII $\left(T \approx 6.3 \times 10^{5}\right)$. It can be clearly seen, therefore, that the speeds we measured for different locations in the coronal holes are subsonic.

\section{Conclusions}

Using the spectral diagnostic capabilities of the SUMER spectrograph, and with a rigorous statistical approach, we have found evidence for propagating magneto-acoustic waves in polar coronal hole regions. For a typical bright enhanced network location the dominant period is $\approx 25 \mathrm{~min}$. Phase measurements were carried out over the full range of frequencies measurable in our data, not just at the dominant period. From these phase measurements, we find evidence that the waves producing the oscillation signatures propagate both upwards and downwards. This is the first time, to our knowledge, that this has been reported in the literature. The dark ("internetwork") areas in pCH show evidence of both upward and downward propagating waves, while bright ("network") areas, by comparison, show only upwardly propagating waves. This may indicate different physical processes occurring in the "internetwork" and "network" regions. From a study of the height variations of the sizes of chromospheric and transition-region features in a small coronal hole and the adjacent quiet Sun, Tian et al. (2008) concluded that loops are much lower in the coronal hole than in the quiet Sun region. From a study of the height variation of the Half Width at Half Maxima of $\left|B_{z} / B\right|$, they further suggested that an open magnetic structure expands through the upper transition region and lower corona more strongly in coronal holes than in the quiet Sun. We may conjecture that the internetwork regions within coronal holes are composed of low-lying coronal loops, where waves can travel both upwardly and downwardly, whereas the network regions are filled with more open, funnel like structures where only upward propagation is possible. Recently, Gomory et al. (2006) reported detection of compressive waves that propagate downward from transition region to the chromosphere in a particular chromospheric network in a quite Sun region. They propose a coronal source of these compressive waves resulting from a nano-flare scenario. In our case clearly we see presence of downward propagation only for internetwork location. One can conjecture that the magnetic topology in quiet sun could be slightly different than in the coronal holes, with presence of more low-lying loop systems in the internetwork regions of coronal loops.

Plotting the phases as histograms, we saw a possible cavity resonance effect on the waves, e.g., $\Delta f$ s of $f / 9$, inferred in reference to Eq. (2), as well as the expected unimpeded propagation of the waves, e.g., a measured phase difference of $360^{\circ}$ between the different parallel lines of phases. We note that the speeds we derived (cf. Table 1) are lower than those found by O'Shea et al. (2007) in polar coronal holes on-disc, and much lower than those found by O'Shea et al. (2006) off-limb. However, the wave speeds measured by O'Shea et al. (2007) were for lines with high formation temperature and, hence, higher up in the atmosphere as compared to our lines. We hypothesise that at lower heights the waves have lower speeds, but the speeds will increase with height in the solar atmosphere. We found the propagation speeds to be consistent with slow magneto-acoustic waves. We should also point out that statistically we find more velocity oscillations than intensity oscillations in this study. For compressional types of waves, we should have signatures in both. So if there are only velocity oscillations present in some locations this implies the presence of transverse-type waves, like Alfvénic. Recent studies from HINODE by De Pontieu et al. (2007) suggest that the chromosphere is permeated by Alfvén waves with strong amplitudes of the order of 10 to $25 \mathrm{~km} \mathrm{~s}^{-1}$ and periods of 100 to 500 s. Tsuneta et al. (2008) have further conjectured that the vertical flux tubes originating from the $\mathrm{kG}$ patches in coronal holes will fan out in the lower atmosphere of the coronal holes 
and will serve as efficient chimney for the propagation of Alfvén waves. These waves may carry enough energy flux for the acceleration of the solar wind. So, it is quite possible that some of these Alfvénic waves have also been detected in our statistical analysis as we see more velocity points as compared to intensity points in the phase plots.

Acknowledgements. Research at Armagh Observatory is grant-aided by the N. Ireland Dept. of Culture, Arts and Leisure. This work was supported by a Royal Society/British Council and STFC grant PP/D001129/1. The SUMER project is financially supported by DLR, CNES, NASA, and the ESA PRODEX programme (Swiss contribution). We thank the Referee for a careful reading of the manuscript and for very valuable suggestions, which has improved the quality of the article.

\section{References}

Athay, R. G., \& White, O. R. 1979, ApJ, 229, 1147

Banerjee, D., O'Shea, E., Doyle, J. G., \& Goossens, M. 2001, A\&A, 380, L39

Dammasch, I. E., Wilhelm, K., Curdt, W., \& Hassler, D. M. 1999, A\&A, 346, 285

De Pontieu, B., McIntosh, S. W., Carlsson, M., et al. 2007, Science, 318, 1574

DeForest, C. E., \& Gurman, J. B. 1998, ApJ, 501, L217

Doyle, J. G., Van Den Oord, G. H. J., O’Shea, E., \& Banerjee, D. 1998, Sol. Phys., 181, 51

Doyle, J. G., Van Den Oord, G. H. J., O’Shea, E., \& Banerjee, D. 1999, A\&A, 347,335
Gomory, P., Rybak, J., Kucera, A., Curdt, W., \& Wohl, H. 2006, A\&A, 448, 1169 Jenkins, G.M., \& Watts, D.G. 1968, Spectral analysis and its applications (Oakland: Holden-Day)

Krieger, A., Timothy, A. F., \& Roelof, E. C. 1973, Sol. Phys., 29, 505

Munro, R. H., \& Withbroe, G. L. 1972, ApJ, 176, 511

Ofman, L., Romoli, M., Poletto, G., Noci, G., \& Kohl, J. L. 1997, ApJ, 491, L111

Ofman, L., Romoli, M., Poletto, G., Noci, G., \& Kohl, J. L. 2000, ApJ, 529, 592

O'Shea, E., Banerjee, D., Doyle, J. G., Fleck, B., \& Murtagh, F. 2001, A\&A, 368,1095

O'Shea, E., Banerjee, D., \& Doyle, J. G. 2006, A\&A, 452, 1059

O'Shea, E., Banerjee, D., \& Doyle, J. G. 2007, A\&A, 463, 713

Popescu, M. D., Doyle, J. G., \& Xia, L. D. 2004, A\&A, 421, 339

Popescu, M. D., Banerjee, D., O’Shea, E., Doyle, J. G., \& Xia, L. D. 2005, A\&A, 442, 1087

Priest, E. R. 1984, Solar Magnetohydrodynamics (Dordrecht: Reidel)

Reeves, E. M. 1976, Sol. Phys., 46, 53

Tian, H., Marsch, E., Tu, C.-Y., Xia, L.-D., \& He, J.-S. 2008, A\&A, 482, 267

Timothy, A. F., Krieger, A. S., \& Vaiana, G. S. 1975, Sol. Phys., 42, 135

Tsuneta, S., et al. 2008 [arXiv: 0807.4631v1]

Torrence, C., \& Compo, G. P. 1998, Bull. Amer. Meteor. Soc., 79, 61

Tu, C.-Yi, Zhou, C., Marsch, E., et al. 2005, Science, 308, 519

Wilhelm, K., Curdt, W., Marsch, E., et al. 1995, Sol. Phys., 162, 189

Wilhelm, K., Dammasch, I. E., Marsch, E., \& Hassler, D. M. 2000, A\&A, 353, 749

Xia, L. D., Marsch, E., \& Curdt, W. 2003, A\&A, 399, L5

Xia, L. D., Marsch, E., \& Wilhelm, K. 2004, A\&A, 424, 1025 